\title{
GRB 110328A/SWIFT J164449.3+573451: THE TIDAL OBLITERATION OF A DEEPLY PLUNGING STAR?
}

\author{
J. K. Cannizzo ${ }^{1,2}$, E. Troja ${ }^{3,5}$, and G. Lodato ${ }^{4}$ \\ ${ }^{1}$ CRESST and Astroparticle Physics Laboratory NASA/GSFC, Greenbelt, MD 20771, USA; John.K.Cannizzo@nasa.gov \\ 2 Department of Physics, University of Maryland, Baltimore County, Baltimore, MD 21250, USA \\ ${ }^{3}$ Astroparticle Physics Laboratory NASA/GSFC, Greenbelt, MD 20771, USA \\ ${ }^{4}$ Dipartimento di Fisica, Università degli Studi di Milano, Milano 20133, Italy \\ Received 2011 May 9; accepted 2011 August 9; published 2011 November 3
}

\begin{abstract}
We examine the tidal disruption event (TDE) scenario to explain Sw 1644+57, a powerful and persistent X-ray source which suddenly became active as GRB 110328A. The precise localization at the center of a $z=0.35$ galaxy argues for activity of the central engine as the underlying cause. We look at the suggestion by Bloom et al. of the possibility of a TDE. We argue that Sw $1644+57$ cannot be explained by the traditional TDE model in which the periastron distance is close to the tidal disruption radius - three independent lines of argument indicate the orbit must be deeply plunging or else the powerful jet we are observing could not be produced. These arguments stem from (1) comparing the early X-ray light curve to the expected theoretical fallback rate, (2) looking at the time of transition to disk-dominated decay, and (3) considering the TDE rate. Due to the extreme excess in the tidal force above that which would be required minimally to disrupt the star in a deeply plunging orbit at periastron, we suggest this scenario might be referred to more descriptively as a tidal obliteration event (TOE) rather than a TDE.
\end{abstract}

Key words: accretion, accretion disks - black hole physics - galaxies: active - galaxies: nuclei

Online-only material: color figures

\section{INTRODUCTION}

Given the massive black holes (MBHs) known to reside at the centers of most galaxies (Magorrian et al. 1998), an inevitable occurrence will be the occasional errant stellar wandering into the inner galactic environment, where the prospect for a tidal disruption and subsequent swallowing of some fraction of the ill-fated star may ensue (Rees 1988, 1990). A complete study of the tidal disruption process was carried out recently by Strubbe \& Quataert (2009, hereafter SQ09) and Lodato \& Rossi (2011, hereafter LR11). For MBHs with greater than $10^{8} M_{\odot}$, the tidal disruption radius lies inside the event horizon for a typical mainsequence (MS) star, so the star would be swallowed whole. The rate of such events is calculated to be $\sim 10^{-5}-10^{-3} \mathrm{yr}^{-1}$ galaxy ${ }^{-1}$, depending on galaxy type (Wang \& Merritt 2004). Wang \& Merritt derive a rate $\sim 10^{-5} \mathrm{yr}^{-1} \mathrm{Mpc}^{-3}$, which, given a local galaxy space density of $\sim 0.01 \mathrm{Mpc}^{-3}$ (Driver et al. 2005), yields an effective rate per galaxy of about $10^{-3} \mathrm{yr}^{-1}$. This is $\sim 10$ times higher than previous estimates (e.g., Cohn $\&$ Kulsrud 1978). The best candidates to date are presented in van Velzen et al. (2010) - two strong examples of optical flares from tidal disruption events (TDEs) in archival Sloan Digital Sky Survey (SDSS) data, from which the authors determine a rate of TDEs $\sim 3_{-2}^{+4} \times 10^{-5} \mathrm{yr}^{-1}$ galaxy $^{-1}$, about a factor of 30 below the Wang \& Merritt theoretical estimate. There were also earlier claims of TDEs (e.g., Komossa et al. 2004, 2008; Gezari et al. 2008, 2009).

After the star comes undone, roughly half of the remnants remain on bound, highly eccentric elliptical orbits and accretes; the other half lies on unbound, hyperbolic orbits and is ejected (Lacy et al. 1982, hereafter LTH82; Rees 1988, 1990). Theoretical research on accretion of tidal debris from tidal disruptions has traditionally been divided into two phases: (1) the immediate unbinding of the star and accretion of streamers of gas,

\footnotetext{
5 NASA Postdoctoral Program.
}

for which a fallback rate of $\dot{M} \widetilde{\propto} t^{-5 / 3}$ is estimated (Rees 1988; Evans \& Kochanek 1989; Lodato et al. 2009; SQ09; RamirezRuiz \& Rosswog 2009; Guillochon et al. 2009; LR11), and (2) the subsequent disk accretion, for which the freely expanding outer disk enforces $\dot{M} \widetilde{\propto} t^{-19 / 16}$ for a thin disk (Cannizzo et al. 1990, hereafter CLG90; Ulmer 1999, SQ09) and $\dot{M} \widetilde{\propto} t^{-4 / 3}$ for a thick disk (Cannizzo \& Gehrels 2009, hereafter CG09). The longer term evolution, which can be cast in terms of accretion disk physics, predicts the tidal disruption flare to be a strong UV source, $\sim 10^{43} \mathrm{erg} \mathrm{s}^{-1}$, which has a duration of months to years. These expectations are nominally satisfied in the handful of secure candidates (van Velzen et al. 2010).

Sw $1644+57$ was triggered by the Swift/BAT as a low signalto-noise ratio $(\mathrm{S} / \mathrm{N})$ image trigger requiring a $2000 \mathrm{~s}$ integration (Cummings et al. 2011; see Burrows et al. 2011 for full details of the Swift analysis). Ground-based NIR and radio observations provided the first clear evidence connecting the X-ray/gammaray source with the galaxy nucleus (Berger et al. 2011). Precise localizations by the Hubble Space Telescope (HST; Fruchter et al. 2011) and Chandra (Levan et al. 2011a) confirmed the transient to lie at the center of its galaxy. Sw $1644+57$ is at a redshift $z=0.3534 \pm 0.0002$ (Levan et al. 2011a, 2011b). The inferred $R$-band magnitude at that distance implies $M_{R} \simeq-18$ (Fruchter et al. 2011), which implies a $\sim 10^{7} M_{\odot} \mathrm{BH}$ at the galactic center, if the galaxy follows the Magorrian relation (Magorrian et al. 1998). Burrows et al. (2011) derive a central $\mathrm{BH}$ mass of $2 \times 10^{7} M_{\odot}$ based on the Magorrian relation, and a lower limit of $\sim 7 \times 10^{6} M_{\odot}$ based on X-ray variability. Quataert \& Kasen (2011) point out, however, that the S/N of the X-Ray Telescope (XRT) data is not sufficient to constrain significant variability on $<10 \mathrm{~s}$ timescales, therefore the central $\mathrm{BH}$ mass could be much lower. Similarly, the host galaxy brightness places only an upper limit on the BH mass from the Magorrian relation, since no "bulge" is actually resolved. In addition, Miller \& Gültekin (2011) use empirical relations relating central BH mass to radio and X-ray luminosity for $\mathrm{Sw} 1644+57$ to argue for 
a central mass of $\log \left(M_{\mathrm{MBH}} / M_{\odot}\right)=5.5 \pm 1.1$. In view of these considerations and the uncertainty associated with applying the Magorrian relation, in this work we seriously entertain the possibility that $M_{\mathrm{MBH}}$ may lie between $\sim 10^{6} \mathrm{M}_{\odot}$ and $\sim 10^{7} \mathrm{M}_{\odot}$.

The positional coincidence gives strong credence to the notion of an event associated with the putative $\mathrm{MBH}$ at the galaxy center, quite probably a TDE (Bloom et al. 2011a, 2011b; Shao et al. 2011), and yet the contrast between theoretical expectation (UV source at $10^{43} \mathrm{erg} \mathrm{s}^{-1}$ ) and observation (gamma-ray/X-ray source at $10^{47}-10^{48} \mathrm{erg} \mathrm{s}^{-1}$ ) lead to the unavoidable conclusion that we are viewing a strongly beamed event. In fact, the jet that we must be observing is completely divorced from the physical properties of the fallback disk, other than relying on accretion as a power supply.

In this work, we estimate the rate of accretion within a transient disk formed from the tidal debris of a disrupted star, and estimate a temperature in the inner disk. We also calculate the expected rates, both for Swift/BAT and all-sky X-ray monitors with $\sim$ mCrab sensitivity.

\section{GENERAL FRAMEWORK}

\subsection{Accretion Properties}

Given the existence of a compact mass $M_{\mathrm{MBH}}$ and the sudden introduction of a much smaller mass of stellar debris $\Delta M$ at $\sim 1-10 R_{S}$, where the Schwarzschild radius $R_{S}=2 G M_{\mathrm{MBH}} / c^{2}$, what will be the subsequent rate of accretion onto the $\mathrm{MBH}$ as a function of time?

In simple physical terms, the disruption of the star occurs when the star comes closer to the $\mathrm{MBH}$ than a tidal disruption radius $R_{T}$ which is determined by demanding that the mean density of the volume of space enclosed by $R_{T}$, i.e., $M_{\mathrm{MBH}} /\left(4 \pi R_{T}^{3} / 3\right)$, be equal to the density of the star. This gives

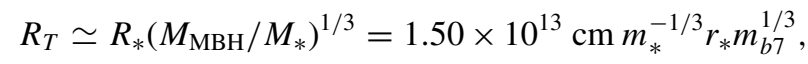

where $m_{*}=M_{*} / M_{\odot}, r_{*}=R_{*} / R_{\odot}$, and $m_{b 7}=M_{\mathrm{MBH}} / 10^{7} M_{\odot}$ (Rees 1988, 1990). Expressing $R_{T}$ in terms of $R_{S}=2.95 \times$ $10^{12} \mathrm{~cm} m_{b 7}$ gives

$$
\frac{R_{T}}{R_{S}}=5.1 m_{*}^{-1 / 3} r_{*} m_{b 7}^{-2 / 3} .
$$

Setting this equal to unity and solving for $m_{b 7}$, we see that for $m_{b 7} \gtrsim 10$, the tidal radius will lie inside the Schwarzschild radius, and an MS star will be swallowed whole. For a maximal Kerr BH in which $R_{S}=G M_{\mathrm{MBH}} / c^{2}$, there would still be another factor of $2^{3 / 2}$ increase in $M_{\mathrm{MBH}}$ before this limit is reached. An equivalent way of visualizing this is in terms of the mean density enclosed by $R_{S}$. For a nonspinning (i.e., Schwarzschild) $\mathrm{BH}$, the mean density out to $R_{S}$ is $\rho_{\mathrm{MBH}}=184 \mathrm{~g} \mathrm{~cm}^{-3} m_{b 7}^{-2}$. For comparison $\rho_{\odot}=1.4 \mathrm{~g} \mathrm{~cm}^{-3}$. Stellar disruption occurs if $\rho_{\mathrm{MBH}}>\rho_{*}$ and $R_{S}<R_{P}<R_{T}$, where $R_{P}$ is the periastron distance of the star.

We define $\xi \equiv R_{P} / R_{T}$. The requirement that the TDE occurs outside the event horizon enforces

$$
\xi>\chi R_{S} / R_{T} \approx 0.2 m_{*}^{1 / 3} r_{*}^{-1} m_{b 7}^{2 / 3} \chi
$$

where $\chi=1$ for a Schwarzschild $\mathrm{BH}$ and $\chi=0.5$ for $\mathrm{a}$ Kerr BH.

We now look at the long-term evolution of the resultant mass accretion rate onto the $\mathrm{MBH}$, both in terms of stellar debris fallback and accretion disk.

\subsubsection{Debris from Stellar Fallback}

Earlier studies give an expression, derived in the Newtonian limit, for the timescale of return of the most bound stellar material to periastron (LTH82; Rees 1988; cf. Equation (4) of LR11):

$$
t_{\text {fallback }} \simeq \frac{\pi}{2^{1 / 2}}\left(\frac{R_{P}}{R_{*}}\right)^{3 / 2} t_{P},
$$

where the dynamical time at periastron $t_{P}=\left(G M_{\mathrm{MBH}} / R_{P}^{3}\right)^{-1 / 2}$. Scaling $R_{P}$ in terms of $R_{T}$ gives

$$
t_{\text {fallback }}=130 \text { days } m_{b 7}^{1 / 2} \xi^{3} m_{*}^{-1} r_{*}^{3 / 2} .
$$

The condition on $\xi$ from Equation (3) gives the lower limit

$$
t_{\text {fallback }}>0.98 \text { days } \chi^{3} m_{b 7}^{5 / 2} r_{*}^{-3 / 2} \text {. }
$$

The accretion rate due to fallback is then (e.g., LTH82; Rees 1988; SQ09; LR11)

$$
\dot{M}_{\text {fallback }} \simeq \frac{1}{3} \frac{M_{*}}{t_{\text {fallback }}}\left(\frac{t}{t_{\text {fallback }}}\right)^{-5 / 3}
$$

so that $\dot{M}_{\text {fallback }} \propto m_{b 7}^{1 / 3} \xi^{2}$. The total fallback mass $\Delta M\left(t_{1}, t_{2}\right)=$ $\int_{t_{1}}^{t_{2}} \dot{M}_{\text {fallback }} d t \rightarrow 0.5 M_{*}$ in the limit $t_{1} \rightarrow t_{\text {fallback }}$ and $t_{2} \rightarrow \infty$. Note that $\dot{M}_{\text {fallback }}=0$ for $t<t_{\text {fallback }}$, and we can thus identify $t=t_{\text {fallback }}$ as the time of the earliest significant BAT activity, $\sim 2$ days before the GRB 110328A trigger.

We can estimate the basic dynamical parameters of the TDE in the following way. Superposed on the long-term X-ray light curve of Sw 1644+57, Levan et al. (2011b) and Bloom et al. (2011b) show a $t^{-5 / 3}$ decay law. This suggests that the X-ray luminosity tracks the rate of return of the fallback material to pericenter. In this work, however, we argue that only the early light curve, $t \lesssim 6$ days, is representative of the stellar debris fallback; the subsequent evolution is dominated by the accretion disk. As mentioned above, the very high X-ray luminosity points to a strongly beamed source, with a beaming factor $\epsilon_{b} \sim 0.01$, so that the intrinsic jet luminosity $L_{\mathrm{j}}$ is related to the observed $L_{X}$ by

$$
L_{\mathrm{j}}=\epsilon_{b} L_{X}
$$

Let us assume that the jet luminosity is a fraction $f$ of the kinetic energy carried by the outflowing material:

$$
L_{\mathrm{j}}=f \dot{M}_{\mathrm{j}} c^{2},
$$

where we have also assumed that the outflow velocity $v \sim c$. The outflow rate itself is a fraction $\epsilon_{j}$ of the fallback rate $\dot{M}_{\text {fallback}}$, where, for a mildly super-Eddington accretion flow, we expect $\epsilon_{j} \sim 0.1$ (SQ09; LR11; Dotan \& Shaviv 2011). We do not wish to restrict ourselves to baryonic jets; this same analysis could be applied to a Poynting flux jet, with $f$ denoting a mass-energy equivalent flux. We can thus obtain an estimate of the fallback rate $\dot{M}_{\text {fallback }}$ as a function of the observed X-ray luminosity:

$$
\dot{M}_{\text {fallback }}(t)=\frac{\epsilon_{b}}{f \epsilon_{j}} \frac{L_{X}(t)}{c^{2}} .
$$

Using Equations (5) and (7), we can thus obtain an estimate of the fallback time and of the penetration factor $\xi$ :

$$
t_{\text {fallback }}=\left(\frac{\epsilon_{b}}{f \epsilon_{j}} \frac{3 L_{X}(t) t}{M_{*} c^{2}}\right)^{3 / 2} t,
$$




$$
\xi=\left(\frac{t}{130 \mathrm{~d}}\right)^{1 / 3}\left(\frac{\epsilon_{b}}{f \epsilon_{j}} \frac{3 L_{X}(t) t}{M_{*} c^{2}}\right)^{1 / 2} m_{*}^{1 / 3} r_{*}^{-1 / 2} m_{b 7}^{-1 / 6}
$$

Note that $\xi$ is very weakly dependent on both the stellar mass and on the BH mass $\xi \propto\left(m_{*} m_{b 7}\right)^{-1 / 6}$ (Krolik \& Piran 2011). We acknowledge the simplicity of the assumption that $f$ and $\epsilon_{j}$ are independent of the Eddington ratio of the accretion rate. There is observational evidence that active galactic nuclei (AGNs) decrease in radio loudness with decreasing Eddington ratio (e.g., Sikora et al. 2007). This may be the result of a fundamental change in the underlying accretion disk structure, e.g., from a slim disk to a thin disk. Based on the total Sw 1644+57 light curve to date, which has exhibited a dynamic variation in averaged X-ray flux of $\lesssim 2$ orders of magnitude, our assumption $\dot{f}=\dot{\epsilon}_{j}=\dot{\epsilon_{b}}=0$ may be at least crudely justified. In other words, although the accretion physics will likely change with Eddington ratio (i.e., slim-to-thin disk), this transition occurs at a low Eddington ratio $\sim 0.01$, which is not applicable to date for Sw $1644+57$.

Several works have considered the observational consequences of ultra-relativistic jets from TDEs prior to Sw 1644+57 (Farrar \& Gruzinov 2009; Giannios \& Metzger 2011). Giannios \& Metzger find, on the assumption of a baryonic jet, that emission from the forward shock may be detectable for several years. However, motivated by results from recent groups studying three-dimensional GRMHD jets (Hawley \& Krolik 2006; McKinney \& Blandford 2009) which find a baryonic zone of exclusion in the jet beam, leading to primarily Poynting flux jets, we do not restrict our focus to baryonic jets, and indeed we view Poynting flux jets to be more likely. On the other hand, the jet luminosity can be dominated by Poynting flux even if most of its rest mass is in baryons.

If the jet remains Poynting dominated out to the distance where it begins to interact with the interstellar medium, a forward shock with concomitant non-thermal emission can still be produced (e.g., Mimica et al. 2009). A highly magnetized jet transfers energy efficiently to the environment, and the Lorentz factor of the forward shock is related to the magnetization of the jet, not its Lorentz factor, which could be much less.

At $t=2.5$ days from the BAT trigger, the X-ray luminosity crosses $10^{47} \mathrm{erg} \mathrm{s}^{-1}$. Inserting these values into Equation (11), after taking into account the offset time of 2 days to be added to the 2.5 days, we obtain $t_{\text {fallback }} \simeq 6480 \mathrm{~s}\left(\epsilon_{b} / f \epsilon_{j}\right)^{3 / 2} \ll 2.5$ days. From Equation (12), we obtain an estimate of $\xi$ :

$\xi \simeq 0.0833\left(\frac{\epsilon_{b}}{0.01}\right)^{1 / 2}\left(\frac{\epsilon_{j}}{0.1}\right)^{-1 / 2}\left(\frac{f}{0.1}\right)^{-1 / 2}\left(m_{*} m_{b 7}\right)^{-1 / 6} r_{*}^{-1 / 2}$.

We thus see that the numbers involved point strongly toward a deeply plunging event with $\xi \ll 1$.

If one assumes $\epsilon_{b}=0.01$ and $\epsilon_{j}=f=0.1$, the resulting $\xi$ value would be smaller than the minimum given by Equation (6). This allows us to put reasonable bounds on the least constrained parameters, $f$ and $\epsilon_{j}$. By requiring that $\xi$ be larger than the minimum value, we obtain

$$
\frac{f \epsilon_{j}}{\epsilon_{b}}<0.18 \chi^{-2 / 3} m_{b 7}^{-5 / 3} r_{*}^{-1} .
$$

Unless $f \epsilon_{j} / \epsilon_{b}$ is much smaller than the value above, the event must have been deeply plunging. Note that the condition above is strongly dependent on the $\mathrm{BH}$ mass. If the $\mathrm{MBH}$ mass were smaller, closer to $10^{6} M_{\odot}$, that would relax the stringency of the deeply plunging constraint.

\subsubsection{Accretion Disk}

The need for a deeply plunging orbit can also be obtained considering the properties of the accretion disk formed by the debris. The process of fallback does not directly supply gas into the $\mathrm{MBH}$, but rather it returns material to $\sim R_{P}$ and therefore feeds an accretion disk. Given the small inferred $t_{\text {fallback }}$ value with respect to the total length of the X-ray light curve, it seems probable that at early times an accretion disk is established. An evaluation of $\int_{t_{\text {fallback }}}^{t_{2}} \dot{M}_{\text {fallback }} d t$ using Equation (7) shows that $90 \%$ of the total available fallback mass $0.5 M_{*}$ has already been supplied to the accretion disk by $t_{2}=32 t_{\text {fallback }}$. It is therefore of interest to estimate the properties of the accretion disk, given a resident disk mass $\sim 0.5 M_{\odot}$.

One may estimate the long-term accretion rate in $\mathrm{Sw} 1644+57$ by looking at the properties of the accretion disk formed from the debris. We idealize the disk as extending from the event horizon to $\eta R_{P}$, where $\eta \simeq 2$. For the high rates of accretion expected, we use the super-Eddington slim disk considered by CG09 (see their Section 2.3.2) in which the radial distribution of surface density $\Sigma(R)$ in a roughly steady disk would be $\propto R^{-1 / 2}$, thus $\Sigma(r)=\Sigma_{0}\left(R / R_{P}\right)^{-1 / 2}$. Therefore, integrating the mass distribution $\int 2 \pi R d R \Sigma(R)$ out to $\eta R_{P}$, and setting it equal to the mass of the fallback debris $\Delta M$, one obtains

$$
\Sigma_{0}=\frac{\Delta M}{(2 / 3) \pi \eta^{2} R_{P}^{2}}=\frac{3}{8 \pi} \frac{\Delta M}{(\eta / 2)^{2} \xi^{2} R_{T}^{2}} .
$$

We derive an estimate for the properties of the resultant disk using algebraic scalings. Inverting Equation (24) of CG09 to obtain $\dot{M}_{0}$ yields

$$
\dot{M}_{0}=1.63 \times 10^{23} \mathrm{~g} \mathrm{~s}^{-1} \Sigma_{0} m_{b 7}^{1 / 2} r_{13}^{1 / 2} \alpha_{-1},
$$

where the disk radius $r_{13}=\eta R_{P} / 10^{13} \mathrm{~cm}$ and $\alpha_{-1}$ is the Shakura $\&$ Sunyaev (1973, hereafter SS73) $\alpha$ parameter in units of 0.1 . Substituting for $\Sigma_{0}$ gives

$$
\dot{M}_{0}=7.72 \times 10^{29} \mathrm{~g} \mathrm{~s}^{-1} \Delta M_{1 / 2} m_{b 7}^{1 / 2} r_{13}^{-3 / 2} \alpha_{-1},
$$

where $\Delta M_{1 / 2}$ is the fallback debris mass in units of $0.5 M_{\odot}$. Substituting for the disk radius $\eta R_{P}$ in units of $10^{13} \mathrm{~cm}$, $r_{13}=1.5 \eta \xi m_{*}^{-1 / 3} r_{*} m_{b 7}^{1 / 3}$, gives

$$
\dot{M}_{0}=4.21 \times 10^{29} \mathrm{~g} \mathrm{~s}^{-1}(\eta / 2)^{-3 / 2} \xi^{-3 / 2} m_{*}^{3 / 2} r_{*}^{-3 / 2} \alpha_{-1} .
$$

Equation (18) only gives a measure of the initial value of the rate of accretion within the accretion disk formed rapidly by stellar fallback. The longer term evolution is given by CG09 as

$$
\dot{M}(t)=\dot{M}_{0}\left(\frac{t}{t_{0}}\right)^{-4 / 3},
$$

where

$$
\begin{aligned}
t_{0} & =\frac{r_{P}^{3 / 2}}{9 / 4 \alpha\left(G M_{\mathrm{MBH}}\right)^{1 / 2}}=\frac{4}{9} \xi^{3 / 2} t_{T} \alpha^{-1} \\
& =224 \mathrm{~s}\left(\frac{\xi}{0.1}\right)^{3 / 2} m_{*}^{-1 / 2} r_{*}^{3 / 2} \alpha_{-1}^{-1},
\end{aligned}
$$

and $t_{T}=\left(G M_{\mathrm{MBH}} / R_{T}^{3}\right)^{-1 / 2}$. The time $t_{0}$ is basically the viscous time for a thick disk at $R_{P}$.

For a disk mass which varies as $t^{-\beta}$, the global viscous timescale $t_{\text {visc }}(t) \equiv\left|M_{\text {disk }} / \dot{M}_{\text {disk }}\right|=\beta^{-1} t$ increases with time. 

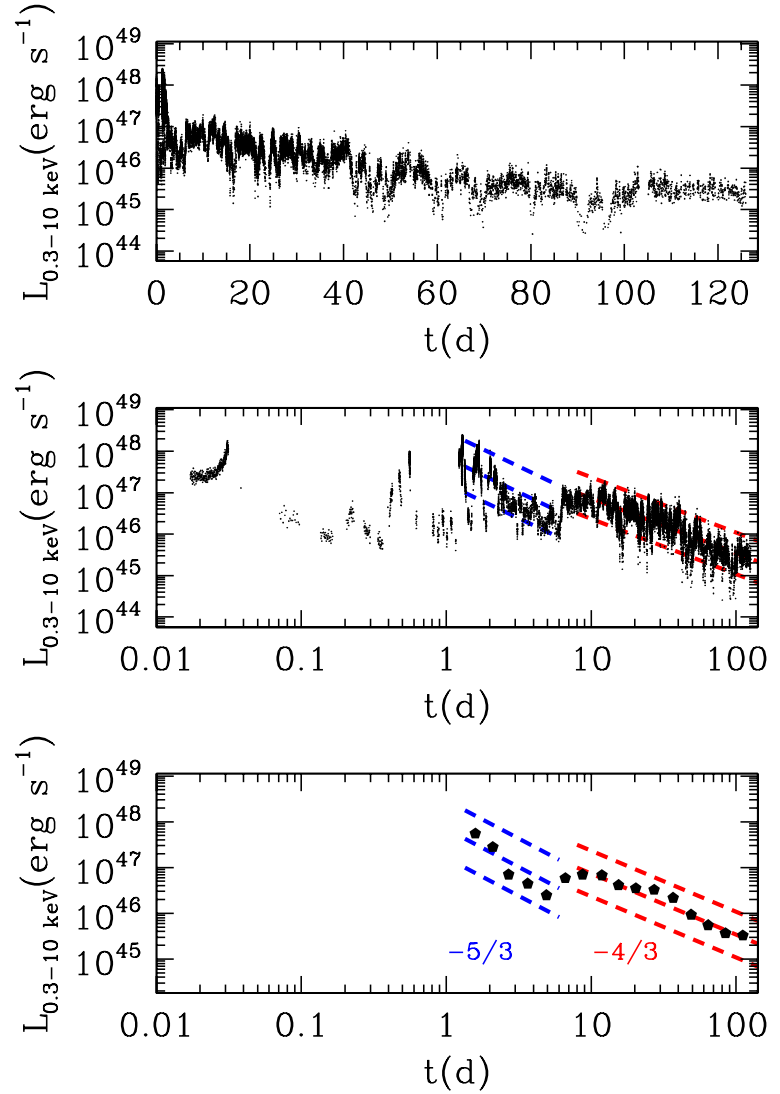

Figure 1. Swift/XRT light curve (Evans et al. 2007, 2009) for Sw 1644+57, plotted on both a log-linear (first panel) and log-log scale (second and third panels). In the third panel, we show the block averages of the X-ray flux, binned in time in 0.125 dex bins. The blue lines indicate the putative stellar debris fallback slope $-5 / 3$ and the red lines show the fallback decline rate expected due to a super-Eddington slim disk, $-4 / 3$. The data do not strongly support either decay law in detail, but are suggestive.

(A color version of this figure is available in the online journal.)

The outer edge of an "external" accretion disk, defined by the lack of a confining torque as from a companion in an interacting binary, moves outward with time. The fact that $t_{\mathrm{visc}}(t) \propto t$ results from the spreading of the outer disk edge, and the viscous time is determined by the slowest timescale in the system, i.e., that at large radii. On the other hand, in comparing the timescales for mass fallback with a viscous timescale, the more relevant time within the disk is not that at the outer edge, but rather the response time of the disk at the radii where the fallback mass is deposited $t_{0}$.

Figure 1 shows the inferred long-term X-ray luminosity for Sw 1644+57, taking into account the distance given by the redshift. The X-ray flux $\sim 10^{47}-10^{48} \mathrm{erg} \mathrm{s}^{-1}$ is super-Eddington for $m_{b 7}=0.1-1$, and also quite different spectrally from theoretical expectations if we were directly observing the disk. On the lower panels, we indicate the expected slopes for stellar debris fallback and accretion due to the external disk formed by the stellar debris. There appears to be a difference in the decay characteristics at $t \simeq 6 \mathrm{~d}$, which we interpret as the start of the disk-dominated decay.

Figure 2 shows a comparison between the stellar debris fallback rate from Equation (7) and the disk accretion rate from Equation (19) for $\xi=0.1$ and 0.2. For $m_{b 7}=1$, the transition point for the $\xi=0.1$ lines $t_{\times}=4.6$ days is close to the time $t \simeq 6$ days in the Sw 1644+57 light curve where there appears to be a change in the decay characteristics. If this is

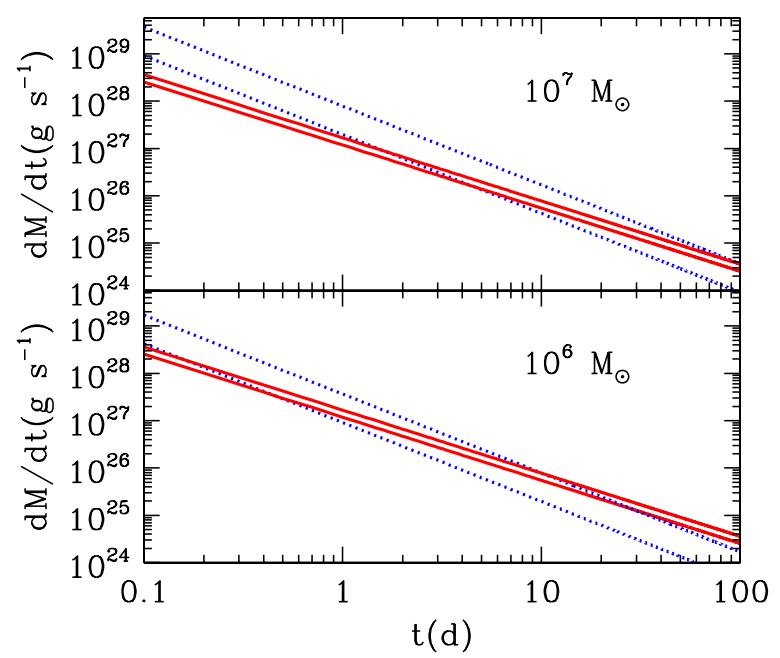

Figure 2. Theoretical accretion rates for stellar debris fallback (blue) and a super-Eddington slim disk (red) for the parameters given in the text. The two sets of lines indicate $\xi=0.1$ and 0.2 . In the upper panel, we take $m_{b 7}=1$ and in the lower panel $m_{b 7}=0.1$. For $m_{b 7}=1$ the transitions lie at $t=4.59$ days $\left(\xi=0.1, t_{\text {fallback }}=3.11 \mathrm{hr}\right)$ and $t=103$ days $\left(\xi=0.2, t_{\text {fallback }}=1.04\right.$ days $)$, with $\xi_{\times}=0.106$ corresponding to $t_{\times}=6$ days; for $m_{b 7}=0.1$ the transitions are at $t=0.46$ days $\left(\xi=0.1, t_{\text {fallback }}=3540 \mathrm{~s}\right)$ and $t=10.3$ days $(\xi=0.2$, $t_{\text {fallback }}=7.87 \mathrm{hr}$ ), with $\xi_{\times}=0.177$ corresponding to $t_{\times}=6$ days.

(A color version of this figure is available in the online journal.)

indeed associated with the debris fallback/disk decay transition, it provides further evidence for a small periastron value $\xi \simeq 0.1$. For $m_{b 7}=0.1$, the putative $t \simeq 6$ day transition corresponds to a larger $\xi \simeq 0.18$. Given the results of Miller \& Gültekin (2011), the latter value may be better motivated. We stress, however, the uncertainties associated with $m_{b 7}$ and $\eta$, and therefore our inferred $\xi$; we certainly do not claim a precise determination of $\xi$. On the other hand, it is noteworthy that the uncertainties in $f, \epsilon_{b}$, and $\epsilon_{j}$ from the previous section do not enter into the $\xi_{\times}\left(t_{\times}\right)$line of argument in this section, and yet the inferred $\xi$ value obtained by adopting reasonable values for $f, \epsilon_{b}$, and $\epsilon_{j}$ and fitting the fallback decay to the early light curve are in line with that obtained using the putative $t_{\times} \simeq 6$ day value in the light curve in conjunction with Equations (7) and (19).

Setting Equations (7) and (19) equal and solving for the time $t_{\times}$defining the transition point yields

$$
t_{\times}=1.25 \times 10^{10} \mathrm{~s} m_{*}^{-3 / 2} r_{*}^{3 / 2} m_{b 7} \alpha_{-1} \xi^{9 / 2}(\eta / 2)^{9 / 2} .
$$

For our favored parameters $m_{*}=r_{*}=\alpha_{-1}=1$, the transition point in the light curve to a disk-dominated decay would lie at

$$
\xi_{\times}=0.106\left(\frac{t_{\times}}{6 \text { days }}\right)^{2 / 9} m_{b 7}^{-2 / 9}\left(\frac{\eta}{2}\right)^{-1} \text {. }
$$

\subsection{Event Rate}

Why have such events not been seen previously? As noted earlier, Sw 1644+57 was nearly missed due to its faintness in the Swift/BAT, which is a $\sim$ mCrab instrument. Its brightness in the Swift/XRT arises because of the XRT's $\sim \mu$ Crab sensitivity.

Swift/BAT rate. The highest $\mathrm{S} / \mathrm{N}$ flare from $\mathrm{Sw} 1644+57$ seen in BAT could have been detected out to $z_{\max } \simeq 0.8^{6}$

\footnotetext{
6 The redshift $z_{\text {det }}=0.35$ for Sw J1644 corresponds to only $\sim 12 \%$ of the estimated sensitivity volume. At present, based on a single detection, it is still premature to speculate on a possible cosmic evolution of TDEs. For the first low-redshift detection of this class of objects-a jetted TDE- the fact that $z_{\text {det }}<z_{\max }$ is most likely an effect of small number statistics (i.e., $N=1$ ). Future observations will shed light on this point. (A second jetted TDE candidate has recently been found with $z=1.2$; Cenko et al. 2011.)
} 
(Burrows et al. 2011), which encompasses a comoving volume of $\sim 90 \mathrm{Gpc}^{3}$. Adopting a local galaxy density $0.01 \mathrm{Mpc}^{-3}$, this gives $\sim 10^{9}$ galaxies in this volume. Taking a nominal tidal disruption rate per galaxy of $3 \times 10^{-5} \mathrm{yr}^{-1}$ (van Velzen et al. 2010) and $\xi \simeq 0.1$, which lowers the nominal rate by a factor of $\sim \xi^{2}$ to $3 \times 10^{-7} \mathrm{yr}^{-1}$ galaxy $^{-1}$, we obtain a net rate $\sim 300 \mathrm{yr}^{-1}$. Adopting a beaming factor $\sim 10^{-2}$ reduces the rate to $\sim 3 \mathrm{yr}^{-1}$, and taking a Swift/BAT sky coverage of $\sim 10 \%$ gives a further reduction to $\sim 0.3 \mathrm{yr}^{-1}$. Note that the standard TDE rate predicts $\sim 30$ BAT events per year (for a beaming factor $\sim 10^{-2}$ ), which can be strongly excluded. This suggests that only a fraction of TDEs can create powerful relativistic jets $(\sim 10 \%$; Burrows et al. 2011) and reinforces the notion of a deep plunging orbit as a way of reducing the overall TDE rate.

Beppo-Sax rate. Beppo-Sax had a field of view (FoV) of $\sim 0.12 \mathrm{sr}$ (FWHM), a sensitivity of $2 \mathrm{mCrab}$ in $100 \mathrm{ks}$, a bandpass of 2-10 keV, and a lifetime of $6 \mathrm{yr}$. Based on the steady state flux observed in XRT, Sw 1644+57 could have been detected up to a redshift $z=1.1$ by the Beppo-Sax/WFCs. Considering the much smaller FoV ( $0.12 \mathrm{sr}$ versus $1 \mathrm{sr}$ of BAT), the rate of events is only 0.2 in $6 \mathrm{yr}$ of the mission, so the fact that Beppo-SAX did not see an event like Sw $1644+57$ is consistent with the BAT rate. By using the brightest peaks, the event could have been observed up to higher redshift $(z \simeq 2.5)$, but it would have been impossible to identify it as a TDE because the long lasting soft emission would have been under detection threshold. In any event, even at $z=2$, the increase in volume does not compensate the difference in FoV.

Wide-field $X$-ray monitor (WFXM) rate. Future mission concepts with sensitive WFXMs envision $\sim 1$ sr sky coverage at $\sim$ mCrab sensitivity. For specificity, we adopt an FoV of $0.6 \mathrm{sr}$, a sensitivity of $1.5 \mathrm{mCrab}$ in $100 \mathrm{~s}$, and a bandpass $0.3-6 \mathrm{keV}$. Using the peak flux $\sim 10^{-8} \mathrm{erg} \mathrm{cm}^{-2} \mathrm{~s}^{-1}$, the event could be detected out to $z \simeq 4$. Based on a BAT detection rate of $1 / 7 \mathrm{yr}^{-1}$, the WFXM rate would be $\sim 1-2 \mathrm{yr}^{-1}$. Currently operating $\mathrm{X}$-ray/ASM instruments such as the RXTE/ASM which has $\sim 50$ mCrab sensitivity in $\sim 6 \times 10^{5}$ s (for blind searches) in the bandpass $2-12 \mathrm{keV}$ would yield much lower rates, therefore their nondetections of these type of events are not unexpected.

\subsection{Falsifiability of the TDE Interpretation}

In the picture of accretion by shredded stellar remnants, $L \propto t^{-5 / 3}$ (Rees 1988), whereas in the accretion disk description $L \propto t^{-4 / 3}$ for a super-Eddington disk with $h / r \simeq 1$ (CG09). The X-ray flux to date has shown a definite decrease, accompanied by large fluctuations. There are indications from comparing the current decay to the early light curve that a transition may have occurred around $t \simeq 6$ days, which we tentatively interpret as the transition to disk decay. Therefore, we should now (as of this writing, at $t \simeq T 0+100$ days) be in the regime for which $d \log F / d \log t \simeq-4 / 3$. The data to date do not definitely substantiate this prediction, but are generally consistent. There are also large variations in the flux which may reflect jet instabilities (McKinney \& Blandford 2009) rather than variations in accretion rate onto the central engine. The present level of X-ray flux is about a factor of $10^{2}$ above the Swift/XRT detection limit, therefore one could envision a more definitive decay law becoming manifest eventually. If no such law emerges or if a different law emerges, that would argue against the TDE interpretation. If on the other hand the flux drops suddenly to an unobservable level, that may be consistent with a sudden change in the properties of the jet as the accretion rate drops below Eddington. Such a change is seen in the radio properties of X-ray binaries as they undergo state changes, e.g., from the high/ thermal state to the low/hard state. For completeness, we note that a third option could be that nonlinear outcome of the TDE is completely different from what we describe, in which case the nondetection of the $t^{-4 / 3}$ law would not be an argument against the TDE. In addition, even though the X-ray flux is currently dominated by the jet, it may be feasible to observe a thermal soft $\mathrm{X}$-ray component from the disk. Using Equation (19), at $t=6$ days our disk model yields a temperature at the innermost stable circular orbit (ISCO) $6 G M_{\mathrm{MBH}} / c^{2}$ of $\sim 0.09 \mathrm{keV} m_{b 7}^{-1 / 2}$, which would produce a thermal component peaking at $\sim 0.2 \mathrm{keV}$ and contributing $\sim 10^{-2}$ in flux to the overall spectrum. According to our estimates, a $\sim 0.1 \mathrm{keV}$ thermal component contributing only $\sim 1 \%$ to the total observed emission could not be detected in the XRT spectra, which are dominated by the jet emission (Burrows et al. 2011).

\section{DISCUSSION AND CONCLUSION}

We have presented simple scalings laws for the post-TDE evolution. Three independent lines of reasoning point to a deeply plunging orbit $R_{P} \ll R_{T}$ if the TDE model is to apply to Sw $1644+57$.

1. An application of the star fallback rate $t^{-5 / 3}$ to the early Sw 1644+57 light curve (as in Levan et al. 2011b) gives $t_{\text {fallback }} \lesssim 1$ day. This implies that the orbit of the disrupted star must have been deeply plunging, $\xi \simeq 0.1$. If $R_{\mathrm{P}} \approx$ $G M_{\mathrm{MBH}} / c^{2}$ the commonly used Newtonian estimate for $t_{\text {fallback }}$ would need correcting (Ayal et al. 2000) because a standard Schwarzschild $\mathrm{BH}$ could not accommodate $\xi<0.2$, for $m_{b 7}=1$. This implies a maximal Kerr BH. Two first-order effects would be Lense-Thirring precession and the general relativistic advance of the lines of apsides of ejecta trajectories (Bardeen et al. 1972; Bardeen \& Petterson 1975). For $m_{b 7}=0.1$, the minimum allowable $\xi$ value (from Equation (3)) would decrease to $\sim 0.04$ for a Schwarzschild $\mathrm{BH}$ and $\sim 0.02$ for a Kerr $\mathrm{BH}$, lessening the stringency imposed by a $\xi \simeq 0.1$ constraint.

2. The long-term $X$-ray luminosity is $\sim 10^{2}-10^{3}$ times greater than expected for Eddington accretion. This points strongly to beaming, as has been inferred for GRBs. In addition, in the long-term light curve there appears to be a change in the decay properties at $t \simeq 6$ days which we interpret as the onset of disk-dominated accretion. By comparing our theoretical decay rates for stellar debris fallback and accretion in a freely expanding super-Eddington disk, we find that a small value $\xi=R_{P} / R_{T}$ is required, $0.1-0.2$, for $M_{\mathrm{MBH}} \simeq 10^{6}-10^{7} M_{\odot}$. The observed X-ray luminosity at $\sim 2.5$ days is $L_{X} / L_{\text {Eddington }} \sim 10^{2}\left(10^{3}\right)$ for $m_{b 7}=1$ (0.1) which would imply a beaming factor of $\epsilon_{b} \simeq 10^{-2}$ (for $f=\epsilon_{j}=0.1$ ), similar to blazars (Sikora et al. 2005; Böttcher et al. 2007). The sudden introduction of $\sim 0.5 M_{\odot}$ of gas inside the ergosphere of a Kerr BH would launch powerful jets within a few local orbital timescales (McKinney 2005). Figure 1 of McKinney shows the steep dependence of jet efficiency on BH spin to mass ratio $J / M$, and in particular a steep upturn close to $J / M \simeq 1$. In fact it may be that our putative constraint on $R_{P}$, namely, $R_{P} \approx G M_{\mathrm{MBH}} / c^{2}$ (which requires $J / M \simeq 1$ ), is a necessary condition for this rare event.

3. The standard TDE rate of $3 \times 10^{-5} \mathrm{yr}^{-1}$ galaxy $^{-1}$ implies a Swift/BAT detection rate of $\sim 30 \mathrm{yr}^{-1}$, for a beaming factor of $\sim 10^{-2}$, which can be strongly excluded. A deeply 
plunging orbit, $\xi \simeq 0.1$, lowers the TDE rate by a factor of $\sim 10^{2}$, giving a rate in line with the BAT detection rate of $\sim 1 / 7 \mathrm{yr}^{-1}$. Our revised rate of $\sim 3 \times 10^{-7} \mathrm{yr}^{-1}$ galaxy $^{-1}$ may need adjusting to correct for relativistic effects. Ayal et al. (2000) compare Newtonian and relativistic TDE methods and find slight differences in $R_{P}$ between the two sets of calculations for the same initial trajectory. They also find that $\sim 75 \%$ of the star becomes unbound, rather than $\sim 50 \%$. However, this depends strongly on the treatment of cooling, which was not modeled in their smoothed particle hydrodynamics (SPH) simulations. Another potential source of concern in our estimates is our use of the SS73-like algebraic scalings for the accretion disk. The SS73 methodology was expanded by Novikov \& Thorne (1973) to include general relativistic corrections including the full Kerr metric. Their multiplicative correction factors are based on Taylor expansions of the form $1+a x+b x^{2}+c x^{3}+\cdots+a^{\prime} y+b^{\prime} y^{2}+c^{\prime} y^{3} \cdots$, where $x=G M_{\mathrm{MBH}} /\left(R c^{2}\right), y=J / M$, and the factors $a, b, c, \ldots$, $a^{\prime}, b^{\prime}, c^{\prime}, \ldots$ are of order unity. These extra multiplicative terms would therefore modify our simple estimates by factors of order unity, even at small radii.

It is worth noting that nearly all previous theoretical studies of tidal disruption have, for simplicity, considered orbits for which the periastron radius $R_{P}$ equals or is slightly interior to $R_{T}$ (Rees 1988; Evans \& Kochanek 1989; CLG90; Ulmer 1999; SQ09). Few works have considered deeply plunging disruptions (e.g., Guillochon et al. 2009). It appears Sw 1644+57 may require $R_{P} \ll R_{T}$, and would be different in at least two important ways from the standard TDE. (1) First, the rates would be lower. The rate for deeply plunging orbits would be less than that for traditional TDEs in which $R_{P} \simeq R_{T}$ by $\sim\left(R_{P} / R_{T}\right)^{2}$, thus giving $\sim 3 \times 10^{-7} \mathrm{yr}^{-1}$ galaxy $^{-1}$, if the more common normal, i.e., "total" TDE rate integrated over all $R_{P}$ values is $\sim 3 \times 10^{-5} \mathrm{yr}^{-1}$ galaxy $^{-1}$. (2) Second, to observe the event we need to be situated within the beam, which would only cover $\sim 10^{-2}$ of the sky. Our estimate of the beam size is based on the ratio of observed X-ray luminosity to expected mass-energy accretion luminosity. There have been no detailed theoretical studies investigating the scenario we outline in this work.

For an orbit in which $R_{P} / R_{T}=0.15$, for example, the tidal force acting on the star at periastron would be $\sim(0.15)^{-3} \simeq 300$ times that minimally needed to unbind the star. The subsequent evolution might be more properly called "obliteration" rather than "disruption," hence we propose the acronym TOE (tidal obliteration event). From SQ09 and earlier studies, in the moving frame of the center of mass of the star, the acceleration of the outer layers due to the $\mathrm{MBH}$ tidal force $\sim\left(G M_{\mathrm{MBH}} / R_{P}^{2}\right)\left(R_{*} / R_{P}\right)$ acting over a dynamical time $t_{P} \simeq\left(G M_{\mathrm{MBH}} / R_{P}^{3}\right)^{-1 / 2}$ results in velocity perturbations to the stellar envelope $\Delta v_{P} \simeq v_{*}\left(R_{T} / R_{P}\right)^{3 / 2}$, where $v_{*}$ is the escape speed from the stellar surface. For $\left(R_{P} / R_{T}\right) \simeq 0.15$, the velocity perturbation exceeds the stellar escape speed by a factor of $\sim 20$. The star would likely be completely shredded in $\sim 10^{2} \mathrm{~s}$. The half of the star remnants with orbits on inward trajectories would immediately establish a super-Eddington disk within the ergosphere of the $\mathrm{MBH}$.

The discovery of a new class of objects raises several interesting questions. For instance, why should a sudden accretion event onto a $\sim 10^{6}-10^{7} M_{\odot} \mathrm{BH}$ in any way resemble a GRB? If there were an accretion event in which $\sim 0.5 M_{\odot}$ were suddenly introduced near the event horizon, the timescales would be consistent with what is observed. Within the first 5 days, the light curve of Sw 1644+57 showed flares with rise and decay times of $\sim 100-200 \mathrm{~s}$. It is unclear whether these variations stem from jet instabilities or are associated with disk timescales. An orbital timescale at the tidal radius $2 \pi / \Omega\left(R_{T}\right)=1.5 \mathrm{hr} r_{13}^{3 / 2} m_{b 7}^{-1 / 2}$ $\left(0.5 \mathrm{hr}\right.$ for $\left.m_{b 7}=0.1\right)$. An after-the-fact examination of prior BAT data revealed a slow brightening over several days, significant at the $\sim 2-2.5 \sigma$ level. The source was already bright in X-rays by the time XRT began observations. It appears we require a deeply plunging orbit, $\xi \simeq 0.1$, and the orbital time $2 \pi r / v_{\phi}$ at the corresponding $R_{P}$ is $\sim 300 \mathrm{~s}$ for $m_{b 7}=1(\sim 100 \mathrm{~s}$ for $\left.m_{b 7}=0.1\right)$. The small periastron radius and general relativistic corrections such as the advance of the line of apsides would enormously speed up the TDE evolution compared to what is shown for instance in Figure 3 of SQ09. This is evidenced in the apparent $\sim 1-10 \mathrm{hr}$ timescale for the fallback time. The process responsible for utilizing the accretion power in the inner disk to tap the BH spin, presumably the magnetorotational instability (Balbus \& Hawley 1998) operating inside the ergosphere (McKinney \& Narayan 2007a, 2007b), would proceed with an initial growth rate given roughly by the local orbital frequency. Given a small seed field within the gas, it is likely that a large number $\gtrsim 30$ of $e$-folding times are required before the shear amplification of the local magnetic field leads to the nonlinear development of a strong jet. Weak precursor events may have contributed to low-level BAT activity preceding the main Sw 1644+57 trigger. Considering the commonalities among jets observed to date in such a wide variety of objects, nature appears to be telling us that the jet physics is largely independent of the mass of the central $\mathrm{BH}$.

We thank L. Piro and E. Rossi for useful conversations. This work made use of data supplied by the UK Swift Science Data Centre at the University of Leicester. E.T. was supported by an appointment to the NASA Postdoctoral Program at the Goddard Space Flight Center, administered by the Oak Ridge Associated Universities through a contract with NASA.

Note added in proof. As of 2011 September 1 the flattening in the Sw 1644+57 light curve evident in Figure 1 beginning at $t \gtrsim 100$ days has continued. A natural physical flux scale is the Eddington value. If the plateau is indeed related to the Eddington flux, and if a super-Eddington slim disk-like state applies to the earlier evolution, it may indicate that the transition to the final state - a thin disk - can occur only after a long period of quasispherical accretion.

\section{REFERENCES}

Ayal, S., Livio, M., \& Piran, T. 2000, ApJ, 545, 772

Balbus, S. A., \& Hawley, J. F. 1998, Rev. Mod. Phys., 70, 1

Bardeen, J. M., \& Petterson, J. A. 1975, ApJ, 195, L65

Bardeen, J. M., Press, W. H., \& Teukolsky, S. A. 1972, ApJ, 178, 347

Berger, E., Levan, A., Tanvir, N. R., et al. 2011, GCN Circ., 11854, 1

Bloom, J. S., Butler, N. R., Cenko, S. B., \& Perley, D. A. 2011a, GCN Circ., 11847,1

Bloom, J. S., Giannios, D., Metzger, B. D., et al. 2011b, Science, 333, 203

Böttcher, M., Basu, S., Joshi, M., et al. 2007, ApJ, 670, 968

Burrows, D. N., Kennea, J. A., Ghisellini, G., et al. 2011, Nature, 476, 421

Cannizzo, J. K., \& Gehrels, N. 2009, ApJ, 700, 1047 (CG09)

Cannizzo, J. K., Lee, H.-M., \& Goodman, J. 1990, ApJ, 351, 38 (CLG90)

Cenko, S. B., Krimm, H. A., Horesh, A., et al. 2011, arXiv:1107.5307v1

Cohn, H., \& Kulsrud, R. M. 1978, ApJ, 226, 1087

Cummings, J. R., Barthelmy, S. D., \& Beardmore, A. P. 2011, GCN Circ., 11823,1

Dotan, C., \& Shaviv, N. J. 2011, MNRAS, 413, 1623

Driver, S. P., Liske, J., Cross, N. J. G., De Propris, R., \& Allen, P. D. 2005, MNRAS, 360, 81 
Evans, C. R., \& Kochanek, C. S. 1989, ApJ, 346, L13

Evans, P. A., Beardmore, A. P., Page, K. L., et al. 2007, A\&A, 469, 379

Evans, P. A., Beardmore, A. P., Page, K. L., et al. 2009, MNRAS, 397, 1177

Farrar, G. R., \& Gruzinov, A. 2009, ApJ, 693, 329

Fruchter, A. F., Misra, K., Graham, J., et al. 2011, GCN Circ., 11881, 1

Gezari, S., Basa, S., Martin, D. C., et al. 2008, ApJ, 676, 944

Gezari, S., Heckman, T., Cenko, S. B., et al. 2009, ApJ, 698, 1367

Giannios, G., \& Metzger, B. D. 2011, arXiv:1102.1429v1

Guillochon, J., Ramirez-Ruiz, E., Rosswog, S., \& Kasen, D. 2009, ApJ, 705, 844

Hawley, J. F., \& Krolik, J. H. 2006, ApJ, 641, 103

Komossa, S., Halpern, J., Schartel, N., et al. 2004, ApJ, 603, L17

Komossa, S., Zhou, H., Wang, T., et al. 2008, ApJ, 678, L13

Krolik, J. H., \& Piran, T. 2011, arXiv:1106.0923v1

Lacy, J. H., Townes, C. H., \& Hollenbach, D. J. 1982, ApJ, 262, 120 (LTH82)

Levan, A. J., Butler, N., Bloom, J., Tanvir, N. R., \& Fruchter, A. S. 2011a, GCN Circ., 11886, 1

Levan, A. J., Tanvir, N. R., Cenko, S. B., et al. 2011b, Science, 333, 199

Lodato, G., King, A. R., \& Pringle, J. E. 2009, MNRAS, 392, 332

Lodato, G., \& Rossi, E. M. 2011, MNRAS, 410, 359 (LR11)

Magorrian, J., Tremaine, S., Richstone, D., et al. 1998, AJ, 115, 2285
McKinney, J. C. 2005, ApJ, 630, L5

McKinney, J. C., \& Blandford, R. D. 2009, MNRAS, 394, L126

McKinney, J. C., \& Narayan, R. 2007a, MNRAS, 375, 513

McKinney, J. C., \& Narayan, R. 2007b, MNRAS, 375, 531

Miller, J. M., \& Gültekin, K. 2011, ApJ, 738, L13

Mimica, P., Giannios, D., \& Aloy, M. A. 2009, A\&A, 494, 879

Novikov, I. D., \& Thorne, K. S. 1973, Black Holes, ed. C. De Witt \& B. De Witt (New York: Gordon \& Breach), 343

Quataert, E., \& Kasen, D. 2011, arXiv:1105.3209v1

Ramirez-Ruiz, E., \& Rosswog, S. 2009, ApJ, 697, L77

Rees, M. J. 1988, Nature, 333, 523

Rees, M. J. 1990, Science, 247, 817

Shakura, N. I., \& Sunyaev, R. A. 1973, A\&A, 24, 337 (SS73)

Shao, L., Zhang, F.-W., Fan, Y.-Z., \& Wei, D.-M. 2011, ApJ, 734, 33

Sikora, M., Begelman, M. C., Madejski, G. M., \& Lasota, J.-P. 2005, ApJ, 625, 72

Sikora, M., Stawarz, Ł., \& Lasota, J.-P. 2007, ApJ, 658, 815

Strubbe, L. E., \& Quataert, E. 2009, MNRAS, 400, 2070 (SQ09)

Ulmer, A. 1999, ApJ, 514, 180

van Velzen, S., Farrar, G. R., Gezari, S., et al. 2010, arXiv:1009.1627v1

Wang, J., \& Merritt, D. 2004, ApJ, 600, 149 